\begin{abstract}
¿Cómo citar este artículo?
De las Heras Martínez, M., Yubero Jiménez, S., y Larrañaga Rubio, E. (septiembre-diciembre, 2020). Cyberbullying en futuros maestros: prevalencia y co-ocurrencia con el bullying en una muestra de estudiantes de magisterio. Revista Virtual Universidad Católica del Norte,
\end{abstract}

(61), 83-101. https://www.doi.org/10.35575/rvucn.n61a6

\title{
| Cyberbullying en futuros maestros: prevalencia y co-ocurrencia con el bullying en una muestra de estudiantes de magisterio
}

\section{Cyberbullying in future teachers: prevalence and co-occurrence with bullying in a simple of pre-service teachers}

\section{Marta de las Heras Martínez}

Estudiante de Doctorado en Investigación en Humanidades, Arte y Educación

Facultad de Educación de Cuenca, Universidad de Castilla- La Mancha

Cuenca, España

marta.heras@uclm.es

Orcid: https://orcid.org/0000-0002-6311-4852

\section{Santiago Yubero Jiménez}

Doctor en Psicología

Facultad de Ciencias de la Educación y

Humanidades de Cuenca, Universidad de

Castilla- La Mancha

Cuenca, España

santiago.yubero@uclm.es

Orcid: https://orcid.org/0000-0002-7148-7958

\author{
Elisa Larrañaga Rubio \\ Doctora en Psicopedagogía \\ Facultad de Trabajo Social de Cuenca, \\ Universidad de Castilla- La Mancha \\ Cuenca, España \\ elisa.larranaga@uclm.es \\ Orcid: https://orcid.org/0000-0002-7183-1683
}

Tipo de artículo: Investigación Científica y Tecnológica.

\section{Resumen}

El bullying es un problema presente en la escuela, ampliándose a formas más novedosas como el cyberbullying. El objetivo de este trabajo fue identificar la prevalencia de ambos fenómenos en los futuros maestros, identificando las variables género y titulación (infantil y primaria), y analizar la relación del comportamiento actual con la participación en etapas previas de la educación, debido al papel central que tendrán en un futuro cercano, por su influencia en la afectividad creada en el aula y por el modelo de aprendizaje ofrecido al alumnado. La muestra estuvo compuesta por 1.122 maestros en formación de la Universidad de Castilla La Mancha (España). Los resultados mostraron tasas de victimización en los estudiantes de magisterio del 57.1 \%, con mayor prevalencia de victimización y agresión en varones y en la titulación de primaria. En las mujeres se relacionó significativamente la victimización y la agresión en primaria con el resto de etapas escolares, mientras que en los hombres la relación sólo se 
estableció entre la etapa de secundaria y la universitaria. La conclusión obtenida refuerza la idea de que el acoso está presente en la universidad, y es necesario trabajar sobre él e influir en la identidad profesional del futuro maestro.

Palabras clave: Bullying; Cyberbullying; Experiencias previas; Futuros maestros; Género; Titulación.

\section{| Abstract}

Bullying is a problem present at school, expanding to newer forms such as cyberbullying. The objective of this study was to identify the prevalence of both phenomena in future teachers, identifying the variables gender and degree (infant and primary education), and to analyze the relationship of the current behavior with the participation in previous stages of the education, due to the central role that they will have in the near future, for their influence on the affectivity created in the classroom and for the learning model that they offer to the students. The sample consisted of 1,122 teachers in training from the University of Castilla La Mancha (Spain). The results showed victimization rates in teaching students of $57.1 \%$, with a higher prevalence of victimization and aggression in boys and in primary school. In women, victimization and aggression in primary education was significantly related to the rest of the school stages, while in men the relationship was only established between the high school stage and the university stage. The conclusion reinforces the idea that bullying is present at the university and the need to work on it and influence the professional identity of the future teacher.

Keywords: Bullying; Cyberbullying; Previous experiences; Pre-service teachers; Gender; Degree.

\section{| Introducción}

Los episodios violentos y el maltrato entre iguales, conocidos como acoso escolar o bullying (Olweus, 1978; Ortega, 2000), ocurren en el ámbito mundial de manera cotidiana en el contexto educativo (Pacheco, 2018) y tienen graves consecuencias sociales (Medina-Cascales y Reverte-Prieto, 2019). En los últimos años, reflejo de la gran trascendencia y auge de las tecnologías de la información y la comunicación (TIC) (Casas et al., 2018; Polino \& Cortassa, 2016), la presentación de la violencia escolar ha derivado en un nuevo fenómeno denominado cyberbullying (P. K. Smith et al., 2008; Tokunaga, 2010), que en la actualidad se encuentra en crecimiento (Pozas-Rivera et al., 2018) y se desarrolla principalmente a través de Internet y el teléfono móvil (Calvete et al., 2010; Garaigordobil y Aliri, 2013). El bullying ha sido conceptualizado desde una perspectiva ecológica, ya que su naturaleza responde a un complejo juego de variables relacionadas con el individuo y con su contexto. Sin duda, la escuela es un espacio de interacciones significativas entre iguales y el comportamiento agresivo se produce en esa interacción (Aronson, 2000). 
El interés de la investigación se centra en la escuela, por su papel fundamental en la protección de cualquier forma de violencia contra las niñas y los niños, ocurra o no en el contexto escolar (Orjuela et al., 2014); y en el maestro, considerado como una figura de gran relevancia para responder a los problemas de acoso (Gorsek \& Cunningham, 2014), concretamente el maestro en formación. El docente asesora a las víctimas (Spears et al., 2015) e interviene como participante de la comunidad educativa dentro de la gestión del aula, con contenidos incluidos en el currículo escolar del centro. Autores como Hombrados et al. (2012) señalan que los alumnos perciben la utilidad de los consejos e información que les proporciona su profesor en la resolución de sus problemas cotidianos. Otros autores afirman que la desaprobación e intervención por parte del profesor en situaciones de acoso hace que disminuya este tipo de problemática en el aula (Saarento, Garandeau et al., 2015; Veenstra et al., 2014). Si los alumnos perciben que el profesor adquiere un compromiso en la prevención del bullying, los problemas de acoso y las peleas entre iguales disminuirán (Espelage et al., 2014; Saarento, Kärnä et al., 2013), beneficiando sobre todo a los alumnos de menor edad y a los que poseen determinadas características personales (Gage et al., 2014).

La coherencia entre el modo de actuar, sentir y pensar es indispensable a la hora de enseñar. La biografía escolar, entendida como el periodo vivido por el futuro maestro siendo este alumno, otorga expresión y significado a lo vivido en sus años como estudiante (Alliaud, 2004), valorando lo aprendido en tanto "le pasó" (como sujeto) por oposición a lo que simplemente "pasó" (Larrosa, 2000). Constituye una fase formativa clave para entender su práctica profesional, en la que la competencia profesional no sólo hará referencia al conocimiento técnico, basado en el saber y el saber hacer, sino también en factores personales vinculados con la toma de decisiones (Esteve y Alsina, 2010), entre los que se encuentra la inteligencia emocional. El estudio aborda aspectos, apenas estudiados en investigaciones previas, en tanto busca analizar la influencia de la escolaridad vivida y su posible participación en dinámicas de acoso en la formación profesional y práctica profesional.

Estudios previos han centrado el análisis del bullying y cyberbullying en la etapa de Educación Secundaria. Sin embargo, investigaciones recientes muestran una alta prevalencia en la etapa de Educación Infantil (Monks y Smith, 2013) y de Educación Primaria (Machimbarrena \& Garaigordobil, 2017), ampliando así el estudio a estos ciclos educativos y resaltando la relevancia del maestro como agente de intervención en las primeras etapas educativas.

Existe también evidencia del acoso escolar en el ámbito universitario, tanto de manera presencial como a través de medios tecnológicos, aunque los estudios del cyberbullying en esta etapa siguen siendo escasos (Méndez et al., 2019; Watts et al., 2017), lo que conlleva a un menor conocimiento de este fenómeno en el contexto de la universidad (J. A. Smith \& Yoon, 2013). La investigación indica que, en esta etapa evolutiva, conocida como "edad adulta emergente" (Arnett, 2004), las características de los individuos están más próximas a los adolescentes que a los adultos (Marini et al., 2006). Es de interés conocer la prevalencia de victimización entre los universitarios para poder llegar a entender mejor el problema de acoso en la edad adulta y establecer programas de prevención e intervención en la universidad. Por otra parte, conocer los comportamientos de acoso en la universidad entre futuros maestros podría ayudar para adecuar la formación de los profesionales de educación en este campo en los programas universitarios. 


\section{Cyberbullying en las etapas de Educación Infantil y Primaria}

El cyberbullying está presente en la etapa de Educación Primaria, tal y como confirman diversos estudios en el plano nacional e internacional (Balakrishnan, 2015; Garaigordobil, 2015; Giménez-Gualdo, 2015; Monks et al., 2016; Navarro et al., 2016; Yang et al., 2014). Medina-Cascales y Reverte-Prieto (2019), afirman que la etapa que comprende de los 6 a los 12 años es la etapa de mayor presencia de acoso escolar. Por su parte, Romera et al. (2019) han mostrado que más del $80 \%$ de los escolares de 6 a 11 años han participado en algún tipo de comportamiento agresivo, siendo las agresiones verbales y relacionales las formas más comunes en los primeros años de escolarización. Resultados similares se han encontrado en otros estudios (García-Fernández et al., 2017; López-Castedo et al., 2018; Zych et al., 2015). Delgado et al. (2014) encontraron en una muestra de 548 estudiantes de 10 a 13 años tasas de participación que alcanzaron el 16,6 \% en víctimas, $6.9 \%$ en acosadores y $31.7 \%$ en observadores. Todos los estudios mencionados apuntan a una mayor incidencia en la preadolescencia, etapa donde la personalidad, los mecanismos decisionales y la consideración de las consecuencias de sus actos no se encuentran consolidados (Márquez-Cervantes y Gaeta-González, 2017). Todo lo indicado señala una mayor relevancia del papel del maestro en esta etapa como agente de intervención.

Los estudios centrados en la etapa de Educación Infantil indican una victimización que difiere en distintos aspectos del bullying de la etapa de Primaria, aunque parece reflejar los origenes de su desarrollo (Monks et al., 2016). Este hecho ha llevado a los investigadores a utilizar términos consensuados como "victimización entre iguales" (Monks et al., 2003; Monks et al., 2005) o "agresividad injustificada" (Ortega y Monks, 2005) para describir estas conductas en la infancia temprana. Los niños entre 4 y 6 años utilizan formas físicas, verbales y relacionales de agresión, pero estas tienden a ser de naturaleza directa (Cerda et al., 2012; Monks et al., 2011). La victimización, además, en etapas iniciales de escolarización se vivencia como una experiencia relativamente breve y de baja estabilidad, lo cual sugiere que un gran número de niñas y niños pueden ser victimizados en la etapa de infantil, aunque es probable que no dure mucho tiempo (Monks y Smith, 2013). Pellegrini et al. (2011) y Roseth et al. (2007) indican que los niños en edad preescolar utilizan la agresión y la conducta prosocial indistintamente.

En las primeras etapas el acoso adopta una forma más sutil, con un mayor número de matices y complejidades que el observado en adolescentes (Adams \& Lawrence, 2011), pero la vulnerabilidad e indefensión están presentes en la víctima igualmente (Romero-Palencia y Plata-Santander, 2015). La constatación de bullying y cyberbullying en las etapas escolares tempranas y medias, con los mismos efectos adversos que en etapas posteriores (García Peña et al., 2013), sugiere la intervención del maestro en la escuela desde las primeras etapas educativas. Principalmente porque una escasa intervención del profesorado reforzaría el acoso y la falta de medidas podría aumentar la percepción de inmunidad por parte de los agresores y la sensación de inseguridad de las víctimas (Šmigelskas et al., 2018).

\section{Cyberbullying en estudiantes universitarios}

Los estudios de cyberbullying en muestras de maestros en el periodo de formación son escasos (Redmond et al., 2018). La mayoría se han centrado en la percepción del acoso por parte de los futuros maestros (Gámex-Guadix et al., 2017; Merma-Molina y Gavilán-Martín, 2019), en la formación recibida (Álvarez-García et al., 2010; Cerezo et al., 2010) y en la capacidad percibida para enfrentar el acoso en las aulas (Álvarez-García et al. 2010; Li, 2008; Redmond et al., 2018; Ríoseco y Menéndez, 2017; Yilmaz, 2010). 
Los estudios de cyberbullying con estudiantes universitarios se iniciaron en Estados Unidos en 2004 con el trabajo pionero de Finn (2004), que informó de una prevalencia entre un 10 \% y un $15 \%$. Los primeros estudios con estudiantes universitarios en España no aparecieron hasta 2015 (Elipe et al., 2015). La prevalencia del cyberbullying en universitarios españoles se sitúan entre el 10 \% (Yubero, Navarro et al., 2017) y el 62,2 \% (Caravaca, Navarro-Zaragoza et al., 2017), dependiendo del instrumento de medida y criterio de clasificación empleado. No hay ninguna investigación específica en España sobre el comportamiento de cyberbullying en los estudiantes de magisterio.

En relación con el género, los resultados de anteriores investigaciones son inconsistentes. Distintos estudios muestran diferencias significativas, indicando una mayor victimización de las mujeres (Faucher et al., 2014; Paullet \& Pinchot, 2014; Zalaquett \& Chatters, 2014); en otros estudios no aparecen diferencias de género (MacDonald \& Roberts-Pittman, 2010); y otros autores han encontrado un mayor rol como agresores en los varones (Hong et al., 2006).

Diversos autores han considerado que el contexto universitario puede proporcionar la continuidad de las conductas del cyberbullying desde la escuela hasta la juventud (Faucher et al., 2014). La investigación ha mostrado que la participación en los procesos de bullying en la etapa de Educación Secundaria tiene continuidad en la universidad (Chapell, et al., 2006; Faucher et al., 2014; Kraft \& Wang, 2010; Paullet \& Pinchot, 2014; Zacchilli \& Valerio, 2011; Zalaquett $\&$ Chatters, 2014). Los estudios realizados con muestras españolas también han informado de la continuidad de los comportamientos de bullying y cyberbullying entre las etapas educativas (Caravaca, Falcón et al., 2016; Yubero, Larrañaga et al., 2017).

Conocer las experiencias previas de los futuros maestros en las etapas iniciales de escolarización, en la etapa de Educación Primaria y Educación Secundaria, a través de datos de la prevalencia en los comportamientos de bullying y cyberbullying, puede ayudar a tomar consciencia de esta realidad, trabajar sobre ella en el contexto universitario e influir en su identidad profesional en un futuro cercano, con el fin de mejorar su intervención educativa y profundizar en su importancia como modelo de aprendizaje en el aula.

El primer objetivo de la presente investigación es conocer la prevalencia del cyberbullying y el bullying en los estudiantes universitarios de magisterio, analizando la influencia del género y de la titulación (Educación Infantil y Educación Primaria). En segundo lugar, analizar la relación de su comportamiento actual con el papel adoptado en las etapas educativas previas, con el fin de comprobar si existe continuidad entre sus comportamientos.

\section{| Método}

\section{Participantes e instrumento}

Los participantes en el estudio fueron 1.122 estudiantes de los 4 cursos académicos del grado de Educación Infantil (548, 48.9 \%) y del grado de Educación Primaria (574, $51.1 \%$ ) de los 4 campus de la Universidad de Castilla-La Macha (España). El 78.3 \% son mujeres y el 21.7 \% son varones, con diferencias significativas en la distribución por género, según el grado (Educación Infantil: 5.5 \% son hombres, y 94.5 \% mujeres; Educación Primaria: 36.4 \% hombres, y 63.6 \% mujeres; $X 2=159.63, p<.001)$. La edad media es de 20.82 años $(D T=3.26)$, sin diferencia significativa entre los grados. 
Se aplicó el cuestionario Bullying Harassment, and Agression Receipt Measure (Hall, 2016); este consta de 14 ítems que describen los comportamientos de bullying (físico, verbal y exclusión social) y de cyberbullying. El alumnado debía informar sobre la frecuencia en que había recibido y/o realizado durante el último mes los diversos comportamientos de acoso de que consta la escala. La fiabilidad en la escala de victimización, según el alfa de Cronbach, es $\alpha=$ .90 , alcanzando fiabilidades elevadas en todas las subescalas: física $\alpha=.78$, verbal $\alpha=.82$, exclusión $\alpha=.80$ y cyberbullying $\alpha=.75$. En la escala de agresión la fiabilidad es $\alpha=.83$, aunque es ligeramente más reducida en alguna subescala, física $\alpha=.75$, verbal $\alpha=.62$, exclusión $\alpha=.61$ y cyberbullying $\alpha=.73$.

Para conocer la experiencia previa del acoso en las etapas de Primaria y Secundaria/Formación Profesional, se utilizó una adaptación del cuestionario de Yubero, Navarro et al. (2017). La medida original incluía 5 ítems relacionados con agresión física directa, agresión verbal directa y exclusión social. Se añadieron 2 ítems más, con contenido cibernético. La fiabilidad fue de $\alpha=.80$ en la escala de victimización y $\alpha=.72$ en la escala de agresión.

\section{Análisis de datos}

En primer lugar, se realizaron los análisis descriptivos de los comportamientos de bullying y cyberbullying en la universidad, tanto para victimización como para agresión. Posteriormente, se analizaron las diferencias en función del género y del grado cursado, empleando MANOVAs y ANOVAs en las variables que resultaron significativas. Por último, se realizó un análisis correlacional para conocer la co-ocurrencia entre los comportamientos de bullying y cyberbullying y su relación con los comportamientos informados retrospectivamente en las etapas previas de educación. Para todos los análisis se utilizó el programa SPSS-24.

\section{| Resultados}

\section{Prevalencia de la victimización y género}

Un $57.1 \%$ de los estudiantes de magisterio informó que había sido víctima en el último mes. Más los hombres (65.1\%) que las mujeres (53.0\%), resultando la diferencia significativa ( $x 2=$ 10.33, p<.001).

En la tabla 1 se encuentran los porcentajes de alumnos que han informado haber sido víctimas según los tipos de bullying. Los hombres han sido, en mayor porcentaje que las mujeres, víctimas de bullying físico y bullying verbal. No se ha encontrado diferencia en las víctimas de cyberbullying. 


\section{Tabla 1}

Porcentajes de victimas según el tipo de bullying y género

\begin{tabular}{llllll}
\hline & Total & Hombres & Mujeres & $\chi^{2}$ & $p$ \\
\hline Fisico & 34.4 & 45.0 & 30.9 & 15.48 & .000 \\
Verbal & 41.9 & 55.5 & 38.2 & 23.81 & .000 \\
Exclusión & 42.0 & 46.2 & 40.7 & 2.48 & .067 \\
Cyberbullying & 11.1 & 10.5 & 11.3 & 0.12 & .415 \\
\hline
\end{tabular}

Nota: Elaboración propia

Victimización, grado y género

Los resultados de los MANOVA (grado x género) ofrecen la comparación de la victimización, atendiendo simultáneamente al género y al grado que están realizando los alumnos. En la tabla 2 se encuentran las medias de victimización agrupadas según las variables de análisis.

Tabla 2

Medias de victimización según grado y género

\begin{tabular}{llllll}
\hline & & Fisica & Verbal & Exclusión & Cyber \\
\hline E. Infantil & Hombre & 0.17 & 0.40 & 0.24 & 0 \\
& Mujer & 0.12 & 0.29 & 0.31 & 0.07 \\
E. Primaria & Hombre & 0.26 & 0.47 & 0.35 & 0.10 \\
& Mujer & 0.17 & 0.37 & 0.38 & 0.09 \\
\hline
\end{tabular}

\section{Nota: Elaboración propia}

Para la victimización física el modelo resultó significativo para la variable género, $F(1,1101)=$ $4.07, p<.05, \eta^{2}=.01$ y para la variable grado, $F(1,1101)=4.91, p<.05, \eta^{2}=.01$, pero no para la interacción género $x$ grado. El análisis univariante mostró mayor victimización en los hombres $(M=0.25, D T=0.36)$ que en las mujeres $(M=0.14, D T=0.29), F(1,1101)=21.22, p<.001, \eta^{2}=.02$. Respecto al grado, el análisis univariante indicó mayor victimización en los alumnos de Educación Primaria $(M=0.20, D T=0.34)$ que en los alumnos de Educación Infantil $(M=0.12, D T=$ $0.27), F(1,1101)=18.71, p<.001, \eta^{2}=.02$. Los hombres presentan mayor victimización física que las mujeres y los alumnos de Educación Primaria mayor victimización física que los alumnos de Educación Infantil.

Para la victimización verbal y la victimización por exclusión los modelos no resultaron significativos en ninguna de las variables de análisis, ni para la interacción género y grado. No se han encontrado diferencias significativas en grado ni en género en los niveles de victimización verbal y victimización por exclusión.

En el modelo del cyberbullying solamente resultó significativo para la interacción, $F(1,1101)=$ $4.21, p<.05, \eta^{2}=.01$. Ningún hombre de Educación Infantil informó recibir comportamientos de cyberbullying. 
Prevalencia de la agresión y género

En la tabla 3 se encuentran los porcentajes de alumnos que han informado haber agredido según los tipos de bullying. Los hombres han sido en mayor porcentaje agresores de bullying físico y bullying verbal. No se ha encontrado diferencia en los agresores de cyberbullying.

\section{Tabla 3}

Porcentajes de agresores según el tipo de bullying y género

\begin{tabular}{llllll}
\hline & Total & Hombres & Mujeres & $\chi 2$ & $p$ \\
\hline Fisico & 20.3 & 39.1 & 15.2 & 66.98 & .000 \\
Verbal & 40.1 & 53.4 & 36.5 & 22.32 & .000 \\
Exclusión & 15.8 & 17.6 & 15.2 & 0.73 & .223 \\
Cyberbullying & 3.9 & 3.8 & 3.9 & 0.01 & .560 \\
\hline
\end{tabular}

Nota: Elaboración propia

Agresión, grado y género

Los resultados de los MANOVA (grado x género) ofrecen la comparación de la agresión, atendiendo simultáneamente al género y al grado que están realizando los alumnos. En la tabla 4 se encuentran las medias de agresión agrupadas según las variables de análisis.

\section{Tabla 4}

\section{Medias de agresión según grado y género}

\begin{tabular}{|c|c|c|c|c|c|}
\hline & & Física & Verbal & Exclusión & Cyber \\
\hline \multirow[t]{2}{*}{ E. Infantil } & Hombre & 0.08 & 0.16 & 0.07 & 0 \\
\hline & Mujer & 0.04 & 0.20 & 0.06 & 0.01 \\
\hline \multirow[t]{2}{*}{ E. Primaria } & Hombre & 0.22 & 0.39 & 0.09 & 0.02 \\
\hline & Mujer & 0.09 & 0.25 & 0.10 & 0.04 \\
\hline
\end{tabular}

\section{Nota: Elaboración propia}

Para la agresión física el modelo resultó significativo para la variable género, $F(1,1088)=$ $11.05, p<.001, \eta^{2}=.01$ y para la variable grado, $F(1,1088)=14.04, p<.001, \eta^{2}=.01$, pero no para la interacción género $x$ grado. El análisis univariante mostró mayor agresión en los hombres $(M=$ $0.20, D T=0.37)$ que en las mujeres $(M=0.06, D T=0.19), F(1,1088)=60.13, p<.001, \eta^{2}=.05$. Respecto al grado, el análisis univariante indicó mayor agresión en los alumnos de Educación Primaria $(M=0.14, D T=0.32)$ que en los alumnos de Educación Infantil $(M=0.04, D T=0.13), F(1,1091)=$ $39.60, p<.001, \eta^{2}=.03$. Los hombres y los estudiantes de Educación Primaria son los grupos que presentan mayor nivel de agresión física entre iguales. 
El modelo de agresión verbal resultó significativo para el grado, $F(1,1102)=11.84, p<.001, \eta^{2}=$ .01 y para la interacción género $x$ grado, $F(1,1102)=4.79, p<.05, \eta^{2}=.01$. El análisis univariante mostró mayor agresión en los estudiantes de Educación Primaria $(M=0.30, D T=0.44)$ que en los estudiantes de Educación Infantil $(M=0.20, D T=0.31), F(1,1105)=19.60, p<.001, \eta^{2}=.02$. Respecto a la interacción, son los estudiantes varones de Educación Primaria los que han informado de mayor nivel de agresión verbal.

Para la agresión por exclusión y la agresión de cyberbullying los modelos no resultaron significativos en ninguna de las variables de análisis, ni para la interacción género y grado. No se han encontrado diferencias significativas en el grado ni en el género en los niveles de agresión por exclusión y agresión a través de nuevas tecnologías.

Co-ocurrencia y género

Para analizar la relación entre el comportamiento actual de bullying y de cyberbullying (victimización y agresión) y el comportamiento previo en dinámicas de acoso, se realizó un análisis correlacional. La tabla 5 muestra correlaciones significativas entre la victimización y la agresión de cyberbullying en la universidad, con un indice menor en las mujeres (hombres= .66, mujeres= .38). También, entre los procesos de bullying y de cyberbullying en la universidad en victimización (hombres $=.57$, mujeres $=.59$ ) y en agresión, menor en los hombres (hombres $=.32$, mujeres=.54). La victimización en la etapa de Primaria se relaciona significativamente con la victimización en las demás etapas en las mujeres. En los hombres se relaciona la victimización de la etapa de Educación Secundaria con la universitaria. Los mismos resultados se han encontrado en la agresión.

\section{Tabla 5}

Correlaciones bivariadas

\begin{tabular}{|c|c|c|c|c|c|c|c|c|}
\hline & 1 & 2 & 3 & 4 & 5 & 6 & 7 & 8 \\
\hline $\begin{array}{l}\text { Victimización } \\
\text { Primaria }\end{array}$ & - & $.31^{* *}$ & .02 & .05 & $.33^{* *}$ & .11 & .05 & .03 \\
\hline $\begin{array}{l}\text { Victimización } \\
\text { Secundaria }\end{array}$ & $.27 * *$ & - & $.30 * *$ & $.41^{* *}$ & .11 & $.40^{* *}$ & $.22 * *$ & $.24^{* *}$ \\
\hline $\begin{array}{l}\text { Victimización } \\
\text { bullying }\end{array}$ & $.17 * *$ & $.29 * *$ & - & $.57^{* * *}$ & .04 & .09 & $.45^{* *}$ & $.27^{* *}$ \\
\hline $\begin{array}{l}\text { Victimización } \\
\text { cyber }\end{array}$ & $.16^{\circ *}$ & $.21 * *$ & $.59 * *$ & - & .12 & $.30^{* *}$ & $.42 * *$ & $.66^{* *}$ \\
\hline Agresión Primaria & $.35^{\circ *}$ & $.12 * *$ & $.22 * *$ & $.20^{* *}$ & - & $.41^{* *}$ & .12 & .11 \\
\hline $\begin{array}{l}\text { Agresión } \\
\text { Secundaria }\end{array}$ & $.10^{* *}$ & .3940 & $.19^{* * *}$ & .06 & $.25 * *$ & - & $.29 * *$ & $.25 * *$ \\
\hline Agresión bullying & $.15^{* *}$ & $.22 * 4$ & $.53^{* *}$ & $.40^{* *}$ & $.35^{* *}$ & $.31^{* * *}$ & & $.32 * *$ \\
\hline Agresión cyber & .03 & $.16^{\circ 6}$ & $.30^{* t}$ & $.38^{* t}$ & $.22^{* *}$ & $.10^{* *}$ & $.54^{* *}$ & - \\
\hline
\end{tabular}

Nota: Elaboración propia. Los valores de los hombres por encima de la línea, los valores de las mujeres por debajo de la línea. ${ }^{*} p<, 05,{ }^{* *} p<, 01$ 


\section{| Discusión}

El primer objetivo del estudio fue conocer la prevalencia del bullying y cyberbullying en una muestra universitaria formada por maestros españoles en formación, analizando la posible influencia del género y grado cursado. Estudios anteriores han encontrado correlación entre la participación en bullying y cyberbullying (Hemphill et al., 2012; Jang et al., 2014), de manera que los alumnos que son agresores de la forma tradicional también pueden serlo mediante las TIC, y las víctimas de bullying también suelen ser víctimas de cyberbullying (MacDonald \& Roberts-Pittman, 2010; P. K. Smith et al. 2008; Sontag et al., 2011; Ybarra \& Mitchell, 2004). Diversas investigaciones plantean que las víctimas, tanto de acoso tradicional como virtual, pueden ser a su vez acosadores cibernéticos (König et al., 2010; P. K. Smith et al., 2008; Sontag et al. 2011; Ybarra \& Mitchell, 2004).

Los resultados de este estudio se han mostrado acordes con la investigación previa, en tanto un $57.1 \%$ de los estudiantes informó que había sido víctima de acoso en el último mes, y un 11.1 $\%$ víctima de cyberbullying. Investigaciones anteriores marcan una elevada prevalencia de conductas de cyberbullying en estudiantes universitarios, con rangos entre 0.22-0.65\% (MacDonald \& Roberts-Pittman, 2010) y entre 10-70 \% (Bottino et al., 2015; Esteve y Alsina, 2010; Modecki et al., 2014; Zych et al., 2016). Las tasas de victimización en población universitaria se encuentran entre el 10-25 \% (Brack \& Caltabiano, 2014; Chapell et al, 2006; Kokkinos et al., 2014; Mateus et al., 2015; Ortega et al., 2008; Paul et al., 2013). En España, los rangos de victimización entre universitarios se sitúan entre un $22.1 \%$ (Dobarro et al., 2018) y un 62.2 \% (Caravaca, Navarro-Zaragoza et al., 2017). No obstante, los resultados de las investigaciones no arrojan datos uniformes que permitan realizar comparaciones entre ellas. Este hecho es debido, fundamentalmente, a tres factores (Yubero, Navarro et al., 2017): en primer lugar, los estudios utilizan diferentes criterios, unos consideran la participación en comportamientos agresivos y otros identifican el sentimiento de participación. En segundo lugar, utilizan diferentes escalas de medida, con diferencias en el número de ítems, desde un único ítem a escalas que incluyen los diversos comportamientos. De hecho, Vandebosch y Van Cleemput (2009) demostraron que si se pregunta sobre los diversos comportamientos se producen más respuestas afirmativas, que si se incluye una pregunta directa sobre la participación en cyberbullying. Y, por último, el periodo de tiempo evaluado en la investigación, pudiendo ser durante toda la vida, el último año o en el momento actual.

En cuanto a la variable género el presente estudio confirma los datos de estudios anteriores que no contemplan diferencias significativas entre hombres y mujeres en el mundo virtual (MacDonald \& Roberts-Pittman, 2010).

El segundo objetivo de esta investigación fue analizar la relación del comportamiento actual del maestro en formación en dinámicas de acoso, con su papel adoptado en etapas previas de su educación, planteándose si existe continuidad en sus comportamientos. Los resultados muestran, en el caso de las mujeres, que ser víctima o actuar como agresora en la etapa de Primaria se relaciona significativamente con la victimización y agresión en el resto de etapas posteriores, mientras que en el caso de los hombres la continuidad, tanto en el papel de víctima como de agresor, sólo existe entre la etapa de Secundaria y la universitaria. Estos datos se encuentran en consonancia con investigaciones previas que constatan la continuidad del bullying de la etapa de Secundaria a la universidad (Chapell, et al., 2006; Kraft \& Wang, 2010; Faucher et al., 2014; Paullet \& Pinchot, 2014; Zacchilli \& Valerio, 2011; Zalaquett \& Chatters, 2014). El presente estudio, además, señala una mayor continuidad en el caso de los varones. 
Asimismo, el estudio se centra en los estudiantes de los grados de Educación de Infantil y Primaria. La investigación científica reciente afirma que la agresión entre iguales aparece en las primeras etapas de escolaridad, siendo en la etapa de Infantil más diádica y menos guiada por un agresor "líder" (Monks et al., 2016). Es menos probable formas indirectas de agresión, lo que puede estar relacionado con capacidades cognitivas más básicas (Björkqvist et al., 1994), niveles más bajos de inteligencia emocional (Kaukianinen et al., 1999), falta de consolidación de su personalidad y mecanismos decisionales, y una menor capacidad para considerar las consecuencias de sus actos (Márquez-Cervantes y Gaeta-González, 2017). Para que aparezcan formas indirectas es necesaria una mayor comprensión de la mente de los otros y ponerse en lugar de los que están a su alrededor (Wellman et al., 2001).

Existe una relación directa y positiva ente bajos niveles de regulación emocional y el empleo de la violencia (Estévez y Jiménez, 2017). Fernández-Berrocal y Extremera (2002) afirman que los maestros son los principales líderes de sus alumnos y es fundamental su formación en inteligencia emocional, ya que las aulas son el modelo de aprendizaje para los discentes, enseñándoles a conocer sus sentimientos, la forma de manejarlos y la capacidad de enfrentarse a los conflictos positivamente (Jiménez-Hernández et al., 2019). En la etapa de la Educación Infantil, la incidencia de los buenos educadores resulta, si cabe, más relevante (Zabalza-Beraza y Zabalza-Cerdeiriña, 2011), sobre todo si se tiene en cuenta que en la etapa de Educación Infantil se persiguen y se cumplen funciones tan importantes como la socialización, la compensación de desigualdades y la optimización del desarrollo del alumno (Belmonte-Almagro et al., 2014). La educación emocional disminuye la prevalencia de factores de riesgo, dotando al sujeto desde edades muy tempranas de herramientas que le permitan afrontar la vida y sus vicisitudes (Gómez Cardona, 2017).

Estas consideraciones remiten a la necesidad de integrar competencias emocionales en el plan de estudios de los estudiantes de Magisterio (Bueno et al., 2005) y a la necesidad de un conocimiento sobre su propia inteligencia emocional como maestros para lograr un buen desempeño laboral (Cejudo y López-Delgado, 2017). La investigación previa con futuros maestros (Cejudo y López- Delgado, 2017; Jiménez-Hernández et al., 2019) ha indicado la necesidad de incluir contenidos relacionados con la gestión emocional de manera transversal en los primeros años de formación académica, y en asignaturas obligatorias como Psicología del Desarrollo o Psicología Educativa. En España, la universidad de Girona ha implementado, en diversas asignaturas del grado de maestro en Educación Infantil y Primaria, distintas prácticas docentes como experiencias de innovación que pueden convertirse en el punto de partida de la formación de futuros docentes en el campo de la inteligencia emocional (Alsina et al., 2016). El primer paso sería la toma de conciencia de sus experiencias previas en la gestión de emociones y las creencias que poseen sobre los conocimientos didácticos y disciplinares necesarios para el oficio de enseñar. Este planteamiento se basa en la idea de que de la reflexión se construye progresivamente el conocimiento profesional y reconoce en la formación de maestros una acción transformadora (Saavedra Bautista, 2018) y se sustenta en el aprendizaje reflexivo como principio general de la formación y se basa en los supuestos de la teoría sociocultural (Vigotsky, 1978) y en la idea del profesional reflexivo (Schön,1983, 1992). También, en la visión realista de la enseñanza (Freudenthal, 1991), según la cual el conocimiento sobre la práctica docente debe ser un conocimiento creado por el mismo sujeto en formación y no un conocimiento ya creado con anterioridad por terceros y transmitido por ellos. Es decir, la persona que se forma lo hace dando significado a unos contenidos más que recibiendo esos contenidos. La formación de educadores emocionalmente inteligentes debe ser uno de los objetivos principales tanto de su formación inicial como continua (Bisquerra et al., 2015). Los futuros docentes tendrán que trabajar siguiendo la premisa de aprendizaje a lo largo de toda la vida, ya que la formación recibida en la etapa universitaria no garantiza un buen desempeño laboral, si no se producen actualizaciones el ncorporaciones de nuevos conocimientos a su práctica en el aula (Montero et 
al., 2017; Veloquio, 2016). Unido a ello, es imprescindible que la gerencia de los centros educativos tome conciencia de la relevancia de las competencias emocionales y del trabajo conjunto comunitario. Como afirman Morgado et al. (2019) si la gerencia no impulsa nuevas formas de educación no será posible una intervención eficaz.

La formación de futuros docentes no debe basarse únicamente en la transmisión de conocimientos o habilidades, sino que debe abarcar aspectos relacionados con el acompañamiento al alumno en su crecimiento personal y en la consolidación de la toma de decisiones. Los factores personales del profesor servirán como modelo para los alumnos de las primeras etapas escolares, con importantes carencias decisionales. La mejor forma de realizar esta tarea es a partir del ejemplo; ya lo decía Séneca (como se citó en Carballo, 2002), "lento es el enseñar por teorías, pero breve y eficaz, por el ejemplo" (p. 4). Los alumnos tenderán a imitarlo, convirtiéndose asi el maestro en una figura influyente, principalmente en la interpretación emocional de situaciones. Del mismo modo puede favorecer la comunicación de su situación al profesorado, por parte de los estudiantes victimizados.

Para concluir, es necesario señalar distintos puntos débiles. La muestra procede únicamente de una universidad, lo cual podría dificultar la generalización de los resultados a la población universitaria española e internacional. No es posible olvidar el efecto de deseabilidad social, siempre que se utilizan auto-informes. Mencionar, igualmente, la sobre-representatividad femenina de la muestra, inherente a los grados universitarios de los ámbitos sociales y educativos.

Se recomienda continuar futuras líneas de investigación con esta muestra particular, que visibilice su notable influencia como modelo de aprendizaje en los escolares, y ayude a tomar conciencia en el contexto universitario de la necesidad de profundizar en su formación e identidad profesional, para afianzar su intervención en el contexto educativo en un futuro cercano, con especial interés en el campo de las relaciones interpersonales, inteligencia emocional y su influencia como modelo de aprendizaje.

\section{| Conclusiones}

Los fenómenos de acoso están presentes en la universidad, en concreto entre los alumnos de las Facultades de Educación. Un 11.1 \% de los estudiantes informó que había sido víctima de cyberbullying en el último mes, casi el 4 \% se identificó como agresor de cyberbullying. No se han encontrado diferencias según el género ni el grado que estaban cursando. Sí han aparecido diferencias significativas en la participación en el bullying, siendo superior en los hombres, tanto en el papel de agresor como de víctima, y en los estudiantes del grado de Educación Primaria.

Por otra parte, el estudio muestra la continuidad del papel adoptado en las dinámicas de acoso entre iguales, con su inicio en las etapas escolares tempranas y con mayor incidencia en el caso de los varones. En el caso de las mujeres, ser víctima o actuar como agresora en la etapa de Primaria se relaciona significativamente con la victimización y agresión en las etapas posteriores, mientras que en el caso de los hombres la continuidad, tanto en el papel de víctima como de agresor, sólo existe entre la etapa de Secundaria y la universitaria. Este aspecto refuerza la importancia del papel que asuma el maestro y su intervención en el aula desde el comienzo de la escolarización. 


\section{| Referencias}

Adams, F. D., \& Lawrence, G. J. (2011). Bullying Victims: The effects last into college. American Secondary Education, 40, 4-13. https://www.jstor.org/stable/23100410

Alliaud, A. (2004). La experiencia escolar de maestros inexpertos. Biografías, trayectorias y práctica profesional. Revista Iberoamericana de Educación, 34(1), 1-11. https://doi.or$\mathrm{g} / 10.35362 /$ rie3412888

Alsina, A., Batllori, R., Falgás, M., Güell, R., y Vidal, I. (2016). ¿Cómo hacer emerger las experiencias previas y creencias de los futuros maestros? Prácticas docentes desde el modelo realista. REDU-Revista de Docencia Universitaria, 14(2), 11-36. https://doi.org/10.4995/redu.2016.5672

Álvarez-García, D., Rodríguez, C., González-Castro, P., Núñez, J. C., y Álvarez, L. (2010). La formación inicial de los futuros maestros en recursos para la convivencia escolar y el manejo del aula. European Journal of Education and Psychology, 3(2),187-198. https://www.redalyc.org/articulo.oa?id=129315468003

Arnett, J. J. (2004). Emerging adulthood: The winding road from the late teens through the twenties. Oxford University Press.

Aronson, E. (2000). Nobody left to hate: Teaching compassion after columbine. Freeman and Co.

Balakrishnan, V. (2015). Cyberbullying among young adults in Malaysia: The roles of gender, age and Internet frequency. Computers in Human Behavior, 46, 149- 157. https://doi.org/10.1016/j.chb.2015.01.021

Belmonte-Almagro, M. L., Lara-Férez, N., Calatayud-Oliver, A. M., y Galián-Nicolás, B. (2014). Familia y escuela ¿dos caras de una misma moneda? En P. Miralles, M. B. Alfageme, y R. A. Rodríguez (Eds.), Investigación e Innovación en Educación Infantil (pp. 91-100). Editum.

Bisquerra, R., Pérez-González, J. C., y García-Navarro, E. (2015). Inteligencia emocional en educación. Síntesis

Björkqvist, K., Lagerspetz, K., \& Österman, K. (1994). Sex differences in covert aggression among adults. Aggressive Behavior, 20, 27-33.

Bottino, S. M., Machado de Campos- Bottino, C., Gómez- Regina, C., \& Villa Lobo- Correira, A. (2015). Cyberbullying and adolescent mental health: systematic review. Cuadernos de Saúde Pública, 31(3), 463-475. https://doi.org/10.1590/0102-311X00036114

Brack, K., \& Caltabiano, N. (2014). Cyberbullying and self-esteem in Australian adults. Cyberpsychology: Journal of Psychosocial Research on Cyberspace, 8(2), 1-10. https://doi.org/10.5817/CP2014-2-7

Bueno, C., Teruel, M. P., y Valero, A. (2005). La inteligencia emocional en alumnos de Magisterio: la percepción y comprensión de los sentimientos y emociones. Revista Interuniversitaria de Formación del Profesorado, 19(3), 169-194.

Calvete, E., Orue, I., Estévez, A., Villardón, L., y Padilla, P. (2010). Cyberbullying en adolescentes: modalidades y perfiles de agresores. Computers in Human Behavior 26(5), 1128-1135.

Caravaca, F., Falcón, M., Navarro-Zaragoza, J., Luna-Ruiz-Cabello, A., Rodriges, O., \& Luna-Maldonado, A. (2016). Prevalence and patterns of traditional bullying victimization and cyber-teasing among college population in Spain. BMC Public Health, 16, 176-185. https://doi.org/10.1186/s12889-016-2857-8

Caravaca, F., Navarro-Zaragoza, J., Luna-Ruiz-Cabello, A., Falcón, M., \& Luna-Maldonado, A. (2017). Association between bullying victimization and substance use among college students in Spain. Adicciones, 29(1), 22-32. https://doi.org/10.20882/adicciones.827

Carballo, R. (2002). Experiencias en grupo e innovación en la docencia universitaria. Complutense. 
Casas, P., Pedreira, M. C., y Romero, L. M. (2018). La televisión como espejo de la realidad del «bullying». Percepciones de los adolescentes sobre el programa "Proyecto Bullying»". Aula Abierta, 47(2), 193-202. https://doi.org/10.17811/rifie.47.2.2018.193-202

Cejudo, J., y López-Delgado, M. L. (2017). Importancia de la inteligencia emocional en la práctica docente: un estudio con maestros. Psicología Educativa, 23, 29-36. https://doi.org/10.1016/j.pse.2016.11.001

Cerda, G., Ortega, R., y Monks, C. (2012). La agresión injustificada como antecedente del bullying: una investigación en Escuelas Infantiles de Chile. Investigación en la Escuela, 78, 55-68. https://doi.org/10.12795/IE.2012.i78.05

Cerezo, F., Ato, M., Rabadán, R., Martínez, F., Orenes, P. J., Calvo, A. R., Ruíz, M., y Bernal, R. M. (2010). La participación familiar en los planes de convivencia escolar. Estudio en los Centros Públicos de Educación Primaria y Secundaria de la Región de Murcia. CARM.

Chapell M. S., Hasselman, S. L., Kitchin, T., Lomon, S. N., Maclver, K. W., \& Sarullo, P. L. (2006). Intimidación en la Escuela Primaria, secundaria y universidad. Adolescencia, 41(164), 633-48.

Delgado, B., Gonzálvez, C., Vicent, M., Gomis, N., e Inglés, C. J. (2014). Artículos e investigaciones de referencia. En M. J. Català, Cultivando Emociones 2 (pp. 230- 279). Generalitat Valenciana.

Dobarro, A., Tuero, E., Bernardo, A. B., Herrero, F. J., y Álvarez-García, D. (2018). Un estudio innovador sobre acoso on-line en estudiantes universitarios. Revista d'Innovació Docent Universitària, 10, 131-142. https://doi.org/10.1344/RIDU2018.10.12

Elipe, P., Mora-Merchan, J. A., Ortega-Ruiz, R., \& Casas, J. A. (2015). Perceived emotional intelligence as a moderator variable between cybervictimization and its emotional impact. Frontiers in Psychology, 6, 486. https://doi.org/10.3389/fpsyg.2015.00486

Espelage, D. L., Polanin, J. R., y Low, S. K. (2014). Percepciones del profesor y del personal del entorno escolar como predictores de la agresión estudiantil, victimización y disposición a intervenir en situaciones de acoso escolar. Psicología Escolar Trimestral, 29(3), 287-305. https://doi.apa.org/doiLanding?doi=10.1037\%2Fspq0000072

Esteve, O., y Alsina, A. (2010). Hacia el desarrollo de la competencia profesional del profesorado. En O. Esteve, K. Melief y Á. Alsina (Eds.), Creando mi profesión. Una propuesta para el desarrollo profesional del profesorado (pp. 7-18). Octaedro.

Estévez, E., y Jiménez, T. I. (2017). Violencia en adolescentes y regulación emocional. International Journal of Developmental and Educational Psychology. Revista INFAD de Psicología, 2(1), 97-104. https://doi.org/10.17060/ijodaep.2017.n1.v2.922

Faucher, C., Jackson, M., \& Cassidy, W. (2014). Cyberbullying among university students: Gendered experiences, impacts, and perspectives. Educational Research International. https://doi.org/10.1155/2014/698545

Fernández-Berrocal, P., y Extremera, N. (2002). La inteligencia emocional como una habilidad esencial en la escuela. Revista Iberoamericana de Educación, 29, 1-6. https://doi.org/10.35362/rie2912869

Finn, J. (2004). A survey of online harassment at a university campus. Journal of Interpersonal Violence, 19, 468-483. https://doi.org/10.1177/0886260503262083

Freudenthal, H. (1991). Revising mathematics education. Kluwer Academic Publishers.

Gage, N. A., Prykanowski, D. A., \& Larson, A. (2014). School climate and bullying victimization: A latent class growth model analysis. School Psychology Quarterly, 29(3), 256-271.

Gámez-Guadix, M., de Santisteban, P., \& Resett, S. A. (2017). Sexting among Spanish adolescents: Prevalence and personality profiles. Psicothema, 29(1), 29-34. 
Garaigordobil, M. (2015). Cyberbullying en adolescentes y jóvenes del País Vasco: cambios con la edad. Anales de Psicología, 31(3), 1069-1077.

Garaigordobil, M., y Aliri, J. (2013). Ciberacoso ("Cyberbullying”) en el País Vasco. Diferencias de sexo en víctima, agresores y observadores. Psicología Conductual, 21(3), 461-474.

García-Fernández, C. M., Romera-Félix, E. M., y Ortega-Ruiz, R. (2017). Cyberbullying en Educación Primaria: Factores explicativos relacionados con los distintos roles de implicación. Psychology, Society, \& Education, 9(2), 251-262.

García Peña, J. J., Moncada-Ortiz, R. M., y Quintero-Gil, J. (2013). El bullying y el suicidio en el escenario universitario. Revista Colombiana de Ciencias Sociales, 4(2), 298-310.

Giménez-Gualdo, A. M. (2015). Cyberbullying. Análisis de su incidencia entre estudiantes y percepción del profesorado (Tesis de doctorado). Universidad de Murcia.

Gómez Cardona, L. (2017). Primera infancia y educación emocional. Revista Virtual Universidad Católica del Norte, 52, 174-184. http://revistavirtual.ucn.edu.co/index.php/RevistaUCN/article/view/950/1397

Gorsek, A. K., \& Cunningham, M. M. (2014). A review of teachers' perceptions and training regarding school bullying. Pure Insights, 3(1), 6. http://digitalcommons.wou.edu/pure/vol3/iss1/6

Hall, W. J. (2016). Initial development and validation of the Bullyharm: The bullying, harassment, and aggression receipt measure. Psychology in the Schools 53(9). http://dx.doi.org/10.1002/pits.21957

Hemphill, S. A., Kotevski, A., Tollit, M., Smith, R., Herrenkohl, T. I., Toumbourou, J. W. \& Catalano, R. F. (2012). Longitudinal predictors of cyber and traditional bullying perpetration in Australian Secondary School students. Journal of Adolescent Health, 51, 59-65. http://dx.doi.org/10.1016/j.jadohealth.2011.11.019

Hombrados, M. I., Gómez, L, Dominguez, J. M., García, P., \& Castro, M. (2012). Types of social support provided by parents, teachers, and classmates during adolescence. Journal of Community Psychology, 40(6), 645-664. https://psycnet.apa.org/doi/10.1002/jcop.20523

Hong, Y., Li, X., Mao, R., \& Stanton, B. (2006). Internet use among Chinese college students: Implications for sex education and HIV prevention. CyberPsychology and Behavior, 10, 161-169. https://doi.org/10.1089/cpb.2006.9973

Jang, H., Song, J. \& Kim, R. (2014). Does the offline bully-victimization influence cyberbullying behavior among youths? Application of general strain theory. Computers in Human Behavior, 31, 85-93. https://doi.org/10.1016/j.chb.2013.10.007

Jiménez- Hernández, D., Sancho-Requena, P., y Sánchez-Fuentes, S. (2019). Perfil del futuro docente: nuevos retos en el marco del EEES. Contextos Educativos, 23, 125-139. http://doi.org/10.18172/con.3471

Kaukiainen, A., Björkqvist, K., Lagerspetz, K., Österman, K., Salmivalli, C., Rothberg, S., \& Anlbom, A. (1999). The relationships between social intelligence, empathy, and three types of aggression. Aggressive Behavior, 25, 81-89.

Kokkinos, C. M., Antoniadou, N., \& Markos, A. (2014). Cyber-bullying: An investigation of the psychological profile of university student participants. Journal of Applied Developmental Psychology, 35(3), 204-214. https://doi.org/10.1016/j.appdev.2014.04.001

König, A., Gollwitzer, M., \& Steffgen, G. (2010). Cyberbullying as an act of revenge? Australian Journal of Guidance \& Counselling, 20(2), 210-224.

Kraft, E., \& Wang, J. (2010). An exploratory study of the cyberbullying and cyberstalking experiences and factors related to victimization of students at a public liberal arts college. International Journal of Technologies, 1, 74-91. http://doi.org/10.4018/jte.2010100106

Larrosa, J. (2000). Pedagogía profana. Estudios sobre lenguaje, subjetividad y formación. Novedades Educativas. 
Li, Q. (2008). Cyberbullying in schools: An examination of preservice teachers' perception. Canadian Journal of Learning and Technology, 34(2), 75-90. https://www.learntechlib.org/p/42831/

López-Castedo, A., Álvarez, D., Domínguez, J., \& Álvarez, E. (2018). Expressions of school violence in adolescence. Psicothema 30, 395-400. http://doi.org/10.7334/psicothema2018.130

MacDonald, C. D., \& Roberts-Pittman, B. (2010). Cyberbullying among college students: Prevalence and demographic differences. Procedia: Social and Behavioral Sciences, 9, 2003-2009. http://doi.org/10.1016/j.sbspro.2010.12.436

Machimbarrena, J. M., \& Garaigordobil, M. (2017). Bullying/Cyberbullying in 5th and 6th grade: differences between public and private schools. Anales de Psicologia, 33(2), 319-326. http://doi.org/10.6018/analesps.33.2.249381

Marini, Z. A., Dane, A. V., Bosacki, S. L., \& Ylc-Cura. (2006). Direct and indirect bully-victims: Differential psychosocial risk factors associated with adolescents involved in bullying and victimization. Aggressive Behavior, 32(6), 551-569. https://doi.org/10.1002/ab.20155

Márquez-Cervantes, M. C., y Gaeta-González, M. L. (2017). Desarrollo de competencias emocionales en preadolescentes: el papel de padres y docentes. Revista Electrónica Interuniversitaria de Formación del Profesorado, 20(2), 221-235. http://doi.org/10.6018/reifop.20.1.232941

Mateus, S., Veiga, A. M., Costa, P., \& das Dores, M. J. (2015). Cyberbullying: The hidden side of college students. Computers in Human Behavior, 43, 167-182. http://hdl.handle.net/10400.26/13944

Medina-Cascales, J. A., y Reverte-Prieto, M. J. (2019). Violencia escolar, rasgos de prevalencia en la victimización individual y grupal en la Educación Obligatoria en España. Revista de Estudios y Experiencias en Educación, 18(3), 97-110. http://doi.org/10.21703/rexe.20191837medina9

Méndez, I., Ruiz-Esteban, C., Martínez, J., y Cerezo, F. (2019). Ciberacoso según características sociodemográficas y académicas en estudiantes universitarios. Revista Española de Pedagogía, 77(273), 261-276. www.jstor.org/stable/26633128

Merma-Molina, G., y Gavilán-Martín, D. (2019). La formación docente a debate: ¿Qué saben los futuros maestros sobre los problemas que afectan la convivencia y el clima del aula? En R. Roig-Vila (Ed.), Investigación e innovación en la Enseñanza Superior. Nuevos contextos, nuevas ideas (pp. 987-997). Octaedro.

Modecki, K., Minchin, J., Harbaugh, A., Guerra, N., \& Runions, K. (2014). Bullying prevalence across contexts: A meta-analysis measuring cyber and traditional bullying. Journal of Adolescent Health, 55(5), 602-611. http://doi.org/10.1016/j.jadohealth.2014.06.007

Monks, C. P., Mahdavi, J. \&, Rix, K. (2016). The emergence of cyberbullying in childhood: Parent and teacher perspective. Psicología Educativa, 22, 39-48.

Monks, C. P., Palermiti, A., Ortega, R., \& Costabile, A. (2011). Peer-victimisation in preschools: a cross-national comparison of England, Spain and Italy. Spanish Journal of Psychology, 14, $129-140$.

Monks, C. P., Smith, P. K., \& Swettenham, J. (2003). Aggressors, victims and defenders in preschool: peer, self and teacher reports. Merrill-Palmer Quarterly, 49, 453-469.

Monks, C. P., Smith, P. K., \& Swettenham, J. (2005). The psychological correlates of peer victimisation in preschool: social cognitive skills, executive function and attachment profiles. Aggressive Behavior, 31, 571-588. https://doi.org/10.1002/ab.20099

Monks, C. P., y Smith, P. K. (2013). El acoso escolar y su prevención. perspectivas internacionales. En A. Ovejero, P. K. Smith y S. Yubero (Coords.), El acoso, la agresión y la victimización en los niños pequeños: Medición, naturaleza y prevención (pp. 91-110). Biblioteca Nueva. 
Montero, L., Colén, M., y Martínez-Piñeiro, E. (2017). Los estudios de Grado en la formación inicial de Maestros en Educación Primaria. Miradas de formadores y futuros maestros. Profesorado. Revista de Curriculum y Formación del Profesorado, 21(1), 1-16. https://www.redalyc.org/articulo.oa?id=56750681001

Morgado, C., Aldana, J., e Isea, J. (2019). Gerencia transformacional desde el accionar docente directivo para el abordaje del Contexto Escolar. Cienciamatria, 5(9), 48-72. https://www.cienciamatriarevista.org.ve/index.php/cm/article/view/99

Navarro, R., Larrañaga, E., \& Yubero, S. (2016). Differences between preadolescent victims and non-victims of cyberbullying in cyber-relationship motives and coping strategies for handling problems whit peers. Current Psychology, 37, 116-127. https://doi.org/10.1007/s12144-016-9495-2

Olweus, D. (1978). Aggression in the schools: Bullies and whipping boys. Hemisphere Press.

Orjuela, L., Cabrera de los Santos, B., Calmaestra, J., Mora-Merchán, J. A., y Ortega, R. (2014). Acoso escolar y Ciberacoso: Propuestas para la acción. Ministerio de Sanidad, Servicios Sociales e Igualdad/Save the Children.

Ortega, R. (2000). Educar la convivencia para prevenir la violencia. Antonio Machado Libros.

Ortega, R., Calmaestra, J., \& Mora-Merchán, J. A. (2008). Cyberbullying. International Journal of Psychology and Psychological Therapy, 8(2), 183-192.

https://www.redalyc.org/articulo.oa?id=56080204

Ortega, R., y Monks, C. P., (2005). Agresividad injustificada entre preescolares. Psicothema 17(3), 453-458.

Pacheco, B. (2018). Violencia escolar: la perspectiva de estudiantes y docentes. Revista Electrónica de Investigación Educativa, 20(1), 112-121. https://doi.org/10.24320/redie.2018.20.1.1523

Paul, L. A., Walsh, K., McCauley, J. L., Ruggiero, K. J., Resnick, H. S., y Kilpatrick, D. G. (2013). Experiencias de mujeres universitarias con divulgación de violación: un estudio nacional. La violencia contra las mujeres, 19(4), 486-502. https://doi.org/10.1177/1077801213487746

Paullet, K., \& Pinchot, J. (2014). Behind the screen where today's bully plays: Perceptions of college students on cyberbullying. Journal of Information Systems Education, 25(1), 63-69. https://aisel.aisnet.org/jise/vol25/iss1/7

Pellegrini, A. D., Van Ryzin, M. J., Roseth, C. J., Bohn-Gettler, C., Dupuis, D., Hickey, M., \& Peshkam, A. (2011). Behavioral and social cognitive processes in preschool children's social dominance. Aggressive Behavior, 37, 248-257. https://doi.org/10.1002/ab.20385

Polino, C., \& Cortassa, C. (2016). Discursos y prácticas de promoción de cultura científica en las políticas públicas de Iberoamérica. Trilogía Ciencia Tecnología Sociedad, 8(15), 13-24. https://doi.org/10.22430/21457778.402

Pozas-Rivera, J., Morales-Reynoso, T., y Martínez-Vilchis, R. (2018). Efectos de un programa de ciberconvivencia en la prevención del cyberbullying. Psychology, Society \& Education, 10(2), 239-250. https://doi.org/10.25115/psye.v10i1.1953

Redmond, P., Lock, J. V., \& Smart, V. (2018). Pre-service teacher's perspectives of ciberbullying. Computer and Education, 119, 1-13. https://doi.org/10.1016/j.compedu.2017.12.004

Ríoseco, M., y Meléndez, D. (2017). Ciberbullying, desde el punto de vista de estudiantes de Pedagogía en una universidad en Chile. Dilemas Contemporáneos: Educación, Política y Valores, 4(2), 1-22.

Romera, E. M., Ortega-Ruiz, R., Rodríguez-Barbero, S., \& Falla, D. (2019). How do you think the victims of bullying feel? A study of moral emotions in Primary School. Frontiers in Psychology, 10, 1753. https://doi.org/10.3389/fpsyg.2019.01753

Romero-Palencia, A., y Plata-Santander, J.V. (2015). Acoso escolar en universidades. Enseñanza e Investigación en Psicología 20(3), 266-274. https://www.redalyc.org/articulo.oa?id=29242800003 
Roseth, C. J., Pellegrini, A. D., Bohn, C., Van Ryzin, M. J., \& Vance, N. (2007). Preschoolers' aggression, affiliation, and social dominance relationships: An observational longitudinal study. Journal of School Psychology, 45, 479-497. https://doi.org/10.1016/j.jsp.2007.02.008

Saarento, S., Garandeau, C. F., \& Salmivalli, C. (2015). Classroom- and school-level contributions to bullying and victimization: A review. Journal of Community \& Applied Social Psychology, 25, 204-218. http://doi.org/10.1002/casp

Saarento, S., Kärnä, A., Hodges, E.V., \& Salmivalli, C. (2013). Student classroom and school level risk factors for vitimization. Journal of School Psychology, 51(3), 421-434. https://doi.org/10.1016/j.jsp.2013.02.002

Saavedra Bautista, C. E. (2018). La formación de maestros en el marco de apuestas tecnológicas emergentes. Revista Virtual Universidad Católica del Norte, (53), 2-17.

Schön, D. A. (1983). The reflective practitioner: How professionals think in action. Basic Books.

Schön, D. A. (1992). La formación de profesionales reflexivos. Hacia un nuevo diseño de la enseñanza y aprendizaje en las profesiones. MEC, Paidós.

Šmigelskas, K., Vaičiūnas, T., Lukoševičiūtè, J., Malinowska-Cieślik, M., Melkumova, M., Movsesyan, E., \& Zaborskis, A. (2018). Sufficient social support as a possible preventive factor against fighting and bullying in school children. International Journal of Environmental Research and Public Health, 15(5), 1-15. http://doi.org/10.3390/ijerph15050870

Smith, J. A. \& Yoon, J. (2013). Cyberbullying presence, extent, and forms in a midwestern post-secondary institution. Information Systems Education Journal, 11, 52-78. http://isedj.org/2013-11/ ISSN: 1545-679X.

Smith, P. K., Mahdavi, J., Carvalho, M., Fisher, S., Russell, S., \& Tippett, N. (2008). Cyberbullying: Its nature and impact in secondary school pupils. Journal of Child Psychology and Psychiatry, 49, 376-385.

Spears, B. A., Campbell, M., Tangen, D., Slee, P. T., \& Cross, D. (2015). Australian pre-service teachers knowledge and understanding of cyberbullying: implications for school climate. Les Dossiers des Sciences de l'Education, 33, 109-130

Sontag, L., Clemans, K., Graber, J., \& Lyndon, S. (2011). Traditional and cyber aggressors and victims: A comparison of psychosocial characteristics, Journal Youth Adolescence, 40, 392-404. https://doi.org/10.1007/s10964-010-9575-9

Tokunaga, R. S. (2010). Following you home from school: A critical review and synthesis of research on cyberbullying victimization. Computers in Human Behavior, 26, 277-287.

http://dx.doi.org/10.1016/j.chb.2009.11.014

Vandebosch, H., \& Van Cleemput, K. (2009). Cyberbullying among youngsters: Profiles of bullies and victims. New Media \& Society, 11, 1349-1371. http://doi.org/10.1177/1461444809341263

Veenstra, R., Lindenberg, S., Huitsing, G., Sainio, M., \& Salmivalli, C. (2014). The role of teachers in bullying: The relation between antibullying attitudes, efficacy, and efforts to reduce bullying. Journal of Educational Psychology, 106(4), 1135-1143. http://doi.org/10.1037/a0036110

Veloquio, G. (2016). La formación permanente de los docentes ante el desafío de atender a la diversidad educativa. Revista Nacional e Internacional de Educación Inclusiva, 9(2),144-154. http://www.revistaeducacioninclusiva.es/index.php/REl/article/viewFile/56/51

Vigotsky, L. S. (1978). Mind in society. The development of higher psychological processes. Harward University Press.

Watts, L. K., Wagner, J., Velasquez, B., \& Behrens, P. I. (2017). Cyberbullying in higher education: A literature review. Computers in Human Behavior, 69, 268-274.

http://dx.doi.org/10.1016/j.chb.2016.12.038

Wellman, H. M., Cross, D., \& Watson, J. (2001). Meta-Analysls of Theory of Mind Development: The truth about false bellef. Chlld Development, 72, 655-684. https://doi.org/10.1111/1467-8624.00304 
Yang, S. C.., Lin, C., \& Chen, A. (2014). A study of Taiwanese teens' traditional and cyberbullying behaviors. Journal of Educational Computing Research, 50(4), 525-552. http://dx.doi.org/10.2190/ec.50.4.e

Ybarra, M. L., \& Mitchell, K. J. (2004) Online aggressor/targets, aggressors, and targets: a comparison of associated youth characteristics, Journal of Child Psychology and Psychiatry, 45(7), 1308-1316. https://doi.org/10.1111/j.1469-7610.2004.00328.x

Yilmaz, H. (2010). An examination of preservice teachers' perceptions of cyberbullying. Eurasia Journal of Mathematics, Science and Technology Education, 6(4), 263-270. https://doi.org/10.12973/ejmste/75248

Yubero, S., Larrañaga, E., \& Navarro, R. (2017). La continuidad del conflicto en la convivencia escolar: medidas de prevención e intervención del acoso. Revista de Paz y Conflictos, 10(2), 89-116. https: //www.redalyc.org/articulo.oa?id=205054523005

Yubero, S., Navarro, R., Elche, M., Larrañaga, E., \& Ovejero, A. (2017). Cyberbullying victimization in higher education: An exploratory analysis of its association with social and emotional factors among Spanish students. Computers in Human Behavior, 75, 439-449. http://dx.doi.org/10.1016/j.chb.2017.05.037

Zabalza-Beraza, M. A., y Zabalza-Cerdeiriña, M.A. (2011). La formación del profesorado de Educación Infantil. CEE Participación Educativa, 16, 103-113.

Zacchilli, T. L., \& Valerio, C. V. (2011). The knowledge and prevalence of cyberbullying in a college sample. Journal of Scientific Psychology, 11-23.

Zalaquett, C. P., \& Chatters, S. J. (2014). Cyberbullying in college: Frequency, characteristic, and practical implications. Sage Open, 4. http://dx.doi.org/10.1177/2158244014526721

Zych, I., Ortega-Ruiz, R., \& Del Rey, R. (2015). Systematic review of theoretical studies on bullying and cyberbullying: facts, knowledge, prevention, and intervention. Aggression and Violent Behavior, 23, 1-21. http://doi.org/10.1016/j.avb.2015. 10.001

Zych, I., Ortega-Ruiz, R., \& Marín-López, I. (2016). Cyberbullying: a systematic review of research, its prevalence and assessment issues in Spanish studies. Psicología Educativa, 22(1), 5-18. https://doi.org/10.1016/j.pse.2016.03.002 